\title{
Machine Replacement, Technology Adoption and Convergence ${ }^{1}$
}

\section{Raouf Boucekkine}

Université Catholique de Louvain and IRES

and

\section{Blanca Martinez}

Université Catholique de Louvain and IRES

December 21, 1999

\begin{abstract}
In this paper, we introduce adoption costs in a canonical vintage capital model. Adoption costs takes the form of a direct loss in production during a fixed period of time. We explicitly characterize the optimal machine replacement policy as a function of the adoption period. Using an explicit numerical method, we study the dynamics of the model. In particular, we find that while an increase in the adoption costs lowers the long run level of output, it also rises the magnitude of short run fluctuations and decreases the convergence speed to the steady states.
\end{abstract}

Keywords: Machine replacement, Technology adoption, Optimal scrapping, Short run fluctuations, Convergence

JEL classification numbers: E22,E32,O40,C63.

\footnotetext{
${ }^{1}$ Correspondence: Raouf Boucekkine, IRES, Université catholique de Louvain, Place Montesquieu,3, 1348 Louvain-la-Neuve, Belgium. e-mail: boucekkine@ires.ucl.ac.be. We thank David de la Croix for helpful discussions.
} 


\section{Introduction}

The recent renewal of the schumpeterian ideas, notably in macroeconomic research, has stimulated a number of alternative approaches to growth and economic fluctuations since the beginning of the nineties. In particular, a number of highly elaborated creative destruction models have been proposed. Among them, Caballero and Hammour's setting (1996) has gained popularity among economists because of its simplicity and the scope of the empirical and theoretical issues that can be addressed within this framework. Indeed, the vintage capital structure adopted by these authors allows to treat quite naturally several crucial issues related to the determinants and consequences of economic obsolescence. ${ }^{2}$

Beside the fact that these models provide an interesting theoretical view of economic fluctuations, one would legitimately question their realism since they are based on a number of extreme assumptions. This paper is designed to relax one of these assumptions, the zero adoption cost assumed in most of the contributions following this line of research. For example, in Caballero and Hammour's model (1996), obsolete production units are replaced without cost by the production units incorporating the most advanced technology. However, there is now a substantial evidence stressing the size of the costs of technology adoption (see for example Jovanovic, 1996). A number of theoretical contributions have already analyzed the role of technology adoption in the growth and development processes, among them the seminal papers of Parente (1994), and Parente and Prescott (1994). In a more recent contribution, Parente (1997) argues in favor of the development theories that have the property that differences in policies translate into differences in steady state income levels rather than in growth rates. And this may be achieved, he adds, by stressing firms' decisions to adopt better technologies subject to the confronted institutional and external constraints. Adoption costs have also been put forward to explain some of the most puzzling stylized facts. For example, Greenwood and Yorokoglu (1997) find that adoption costs (through learning and training) may well have caused the productivity slowdown observed after the first oil shock.

In this paper, we use a very simplified version of the creative destruction model due to Caballero and Hammour (1996). Adoption costs are modeled as a direct output loss during a fixed period of time, say the adoption period. By proceeding in this way, our specifications are rather consistent with the institutional barriers view of adoption advocated by Parente and Prescott (1994). We derive the optimal machine replacement policy as a function of the adoption costs. It is known that the average age of capital is markedly higher in developing countries and that adoption costs may well account for this. Our simple model is good at depicting with fine details the relationship between adoption costs and optimal scrapping rules. Following Parente

\footnotetext{
${ }^{2}$ Benhabib and Rustichini, 1991, Boucekkine, Germain and Licandro, 1997, Boucekkine, del Río and Licandro, 1999, and Greenwood and Yorokoglu, 1997, are four other illustrations of this kind of settings.
} 
(1997), we also study how adoption costs affects the long run value of output level. Although the latter effects turn out to be much complex than expected, we find that in general there exists a negative correlation between adoption costs and long run output levels. Finally, using an explicit numerical method described by Boucekkine, Licandro and Paul (1997), we find that the transition to the long run equilibrium is unsurprisingly oscillatory due to the well-known "replacement echoes" mechanism inherent to vintage models as outlined by Benhabib and Rustichini (1991). Adoption costs are finally shown to increase the magnitude of fluctuations and so to lower the speed of convergence.

This paper is organized as follows. Section 2 presents briefly the model. Section 3 characterizes the optimal machine replacement policy and the long run output level. Section 4 analyzes short run fluctuations using an explicit numerical algorithm. Section 5 concludes.

\section{The model}

We consider an economy with vintage capital and Leontieff technology as in Caballero and Hammour (1996). Technological progress is exogenous and we assume that it is fully embodied in the newest capital goods. Given a constant rate of technical progress $\gamma$, a unit of capital of vintage $t$ requires $e^{-\gamma t}$ units of labor. Hence the old vintages become less and less efficient with respect to the newest vintages. We assume that our economy does not innovate, it simply adopts the most advanced technologies. However, adoption is costly as it involves a direct loss of production during say the adoption period $D$. Production and labor demand can be written respectively as:

$$
\begin{aligned}
Y(t) & =\int_{t-T(t)}^{t} H(s) e^{\gamma s} d s-\int_{t-D}^{t} \alpha H(s) e^{\gamma s} d s, \\
L(t) & =\int_{t-T(t)}^{t} H(s) d s .
\end{aligned}
$$

$H(t)$ denotes the hiring rate of the economy and $T(t)$ denotes the oldest production unit still operated at $t$ (or scrapping time). Adoption costs are introduced through two parameters, $D$ the adoption period and $\alpha, 0<\alpha<1$, the fraction of output lost during $D$. In our model, $T(t)$ is determined endogenously in the model in contrast to the adoption period $D$ which is taken constant. The assumptions underlying our specifications are obviously consistent with $T(t)>D$ for every $t$. This technical issue is addressed later on.

The rest of the model is completely standard. In particular, we assume that consumers have linear utility functions, that labor supply is inelastic, equal to 1 , and that hirings are determined by a generalized Nash bargaining between workers and entrepreneurs exactly as in Caballero and Hammour like models. Since the 
investment required to create a production unit is specific to this unit, the asset values of outside options is lower with respect to the inside option for both workers and entrepreneurs. This fact introduces a rigidity in the labor market: Workers and entrepreneurs will form a bilateral monopoly since both prefer to preserve the match. Denote by $U(t)$ the resulting unemployment rate. With our previous assumptions and notations:

$$
U(t)=1-\int_{t-T(t)}^{t} H(s) d s
$$

Typically workers and entrepreneurs will bargain over an appropriable surplus $S(t)$. The surplus is equal to the present value of production over the maximum planned lifetime, say $J(t)$, minus the entrepreneurs and workers outside options. Assuming that the entrepreneurs cannot recover the investment costs, their outside option is zero. As for workers, the outside alternative is to turn unemployed and search for another job. Under perfect foresight, we have necessarily

$$
J(t)=T(t+J(t)) .
$$

We assume a generalized Nash-bargaining solution, with a share $\beta \in(0,1)$ of the surplus going to the worker and $1-\beta$ going to the firm. Under these assumptions, the surplus associated to a job and the corresponding Nash-bargaining equilibrium conditions are:

$$
\begin{gathered}
S(t)=\int_{t}^{t+J(t)}\left[e^{\gamma t}-\varpi(s) e^{\gamma s}\right] e^{-r(s-t)} d s-\alpha \int_{t}^{t+D} e^{\gamma t} e^{-r(s-t)} d s \\
\varpi(t) e^{\gamma t}=\beta \frac{H(t)}{U(t)} S(t), \\
e^{\gamma t}=(1-\beta) S(t) .
\end{gathered}
$$

Equation (6) stipulates that the outside option wage $\varpi(t) e^{\gamma t}$ is equal to the flow of probability of finding a job times the fraction of the surplus going to the worker. Equation (7) states that on the firm's side, the cost of creating a production unit $e^{\gamma t}$ must be equal to the firm's share of the appropriable surplus. Finally, the expected lifetime $J(t)$ is determined by the exit condition:

$$
e^{\gamma t}=\varpi(t+J(t)) e^{\gamma(t+J(t))} .
$$

A job will be destroyed when its productivity is lower than the worker's outside option. Combining the exit condition with (7)-(5) and with (6)-(5) respectively, 
yields the following conditions:

$$
\begin{aligned}
\frac{1}{1-\beta} & =\int_{t}^{t+J(t)}\left[1-e^{-\gamma(t-s+T(s))}\right] e^{-r(s-t)} d s-\alpha \int_{t}^{t+D} e^{-r(s-t)} d s, \\
e^{-\gamma T(t)} & =\frac{\beta}{1-\beta} \frac{H(t)}{U(t)} .
\end{aligned}
$$

We are now able to define an equilibrium for our economy. Given initial conditions $H(t) \geq 0, \forall t<0$, an equilibrium for this economy is a path $\{H(t), Y(t), U(t), T(t)$, $J(t)\}, \forall t \geq 0$, that solves the equations (1), (3), (4), (8), and (9), provided $T(t)>D$ and $0 \leq U(t) \leq 1$ for all $t \geq 0$. The next two sections characterize the equilibrium paths of the model.

\section{Optimal scrapping and long run output level}

\subsection{Optimal scrapping and adoption costs}

As usual in this type of vintage models (see for example Boucekkine, Germain and Licandro, 1997), one has to first solve for the timing variables $T(t)$ and $J(t)$ which appear separately in the purely forward-looking sub-block (4)-(8). Differentiating

(8) and rearranging terms, we get

$$
T(t)=G(J(t))=-\frac{1}{\gamma} \ln \left[1-\frac{r-\gamma}{1-\beta}-\frac{\gamma}{r}\left(1-e^{-r J(t)}\right)-\alpha\left(1-e^{-r D}\right)\left(1-\frac{\gamma}{r}\right)\right] .
$$

Function $G($.$) is defined on R_{+}$and returns values in $R_{+}$under the following sufficient and necessary conditions

Assumption 1 The parameters must check the following conditions:

$$
\begin{aligned}
& 0<\gamma<r \\
& r<(1-\beta)\left[1-\alpha\left(1-e^{-r D}\right)\right]
\end{aligned}
$$

The first condition is standard in growth models and ensures that utility is bounded in the steady state. The second condition implies actually an upper bound for the adoption period $D$. As argued above, our problem only makes sense if $T(t)>D$. The second assumption is consistent with this requirement but it does not ensure its fulfillment as we will see later. Note that under Assumption 1, function $G($.$) is increasing and admits a unique strictly positive fixed-point, say T_{G}$. We are now able to characterize the optimal scrapping policy.

Proposition 1 Under Assumption 1: (i) $T(t)=J(t)=T^{\star}=T_{G}, \forall t \geq 0$. (ii) $T^{\star}=T^{\star}(D)$ is an increasing and concave function of the adoption period $D$. 
Proof: The proof of Property (i) is identical to the proof of Proposition 2 in Boucekkine, Germain and Licandro (1997). Since function $G($.$) is increasing and$ since it admits a unique positive fixed-point, the optimal scrapping rule is constant, equal to this fixed-point. On the other hand, as $\frac{\partial T^{*}}{\partial D}=\frac{(r-\gamma) \alpha e^{-r D}}{\gamma\left(e^{-\gamma T^{*}}-e^{-r T^{*}}\right)}>0, T^{\star}$ is an increasing function of the adoption period. ${ }^{3}$ After tedious computations, one can also establish that the second derivative $\left(T^{\star}\right)^{\prime \prime}(D)$ has the reverse sign of the expression

$$
\alpha^{2} e^{-2 r D} \gamma e^{-r T^{*}}+\left[\frac{\alpha r e^{-r D} e^{-\gamma T^{*}}\left(1-e^{-(r-\gamma) T^{*}}\right)}{r-\gamma}\right] *\left[\frac{e^{-\gamma T^{*}}-e^{-r T^{*}}}{r-\gamma}-\frac{\alpha e^{-r D}}{r}\right] .
$$

As by construction of function $G($.

$$
\frac{e^{-\gamma T^{*}}-e^{-r T^{*}}}{r-\gamma}-\frac{\alpha e^{-r D}}{r}=\frac{1-e^{-r D}-\alpha}{r}-\frac{1}{1-\beta}
$$

it turns out that $T^{\star}$ is a concave function of $D$ if $r<(1-\beta)\left[1-\alpha-e^{-r D}\right]$, which is ensured by Assumption 1.

In our model, adoption costs increase the lifetime of production units and delay the replacement of the oldest machines. This is a very good property of our model since adoption costs have been repeatedly invoked to explain technological sclerosis and the (markedly) higher age of capital in developing countries (see Jovanovic, 1996, again). As the relationship is also concave, we can establish the following existence result.

Proposition 2 Under Assumption 1: (i) There exists $D_{0}>0$ such that $T^{\star}>D$ if and only if $0 \leq D<D_{0}$. (ii) For any initial conditions $H(t), t<0$, such that $\int_{-T^{\star}}^{0} H(\tau) d \tau<1$, then $0<U(t)<1 \forall t \geq 0$.

The previous proposition provides the conditions on the parameters under which $T(t)=T^{\star}$ is an equilibrium for all $t \geq 0$. Condition (i) is a direct consequence of the concavity property proved in Proposition 1. For $r=0.05, \gamma=0.03$ and $\beta=0.5, T^{\star}$ equals to 14.61 years, for $D=1$ year and $\alpha=20 \%$, while $D_{0}$ is around 24 years. We are obviously thinking of adoption periods of a few years at most to fit institutional barriers to adoption as import licensing and the like; and in this context, condition (i) works quite well. Condition (ii) ensures that the requirement $0 \leq U(t) \leq 1$ is met for all $t \geq 0$ when the interior solution $T^{\star}$ is implemented from $t=0$. Clearly, huge hiring rates in the past would yield a negative unemployment rate at $t=0$ by (3) if $T(0)$, being fixed to $T^{\star}$, is not allowed to adjust. If this situation is prevented at $\mathrm{t}=0$ (by the condition $\int_{-T^{\star}}^{0} H(\tau) d \tau<1$ ), it will never

\footnotetext{
3 The same qualitative results can be established for the other cost parameter $\alpha$.
} 
happen in the future (See Boucekkine, Del Río and Licandro, 1999, Proposition 4, for a proof of the latter claim).

\subsection{Some properties of the steady state growth paths}

In the steady state, output should increase at the exogenous rate of technical progress $\gamma, Y(t)=Y e^{\gamma t}, Y>0$. We denote by $H$ the long run hiring rate. Solving the model in the steady state yields:

$$
\begin{aligned}
H & =\frac{\frac{1-\beta}{\beta} e^{-\gamma T^{*}}}{1+\frac{1-\beta}{\beta} e^{-\gamma T^{*} T^{*}}} \\
Y & =\frac{H}{\gamma}\left[1-e^{-\gamma T^{*}}-\alpha\left(1-e^{-\gamma D}\right)\right] .
\end{aligned}
$$

It follows that an increase in the adoption period decreases the hiring rate through a rise in the lifetime of the production units. Indeed

$$
\frac{\partial H}{\partial T^{*}}=-\frac{\left(\frac{1-\beta}{\beta} e^{-\gamma T^{*}}\right)\left(\gamma+\frac{1-\beta}{\beta} e^{-\gamma T^{*}}\right)}{\left(1+\frac{1-\beta}{\beta} e^{-\gamma T^{*}} T^{*}\right)^{2}}<0 .
$$

Since a rise in $T^{\star}$ decreases both the probability of finding a job and the separation rate, the effect on job creation is unambiguously negative. As an increase in the adoption period rises the optimal scrapping time by Proposition 1, it turns out that investment is depressed if the adoption period is lengthened. Things are more complex for the long run output level. Indeed, we have

$$
\frac{\partial Y}{\partial D}=\frac{Y}{H} \frac{\partial H}{\partial D}+H e^{-\gamma T^{*}} \frac{\partial T^{*}}{\partial D}-\alpha H e^{-\gamma D}
$$

Equation (10) shows clearly the three effects at work in the comparative statics of long run output level. The first one is negative as argued just above. The second one is positive: An increase in the adoption period increases the lifetime of machines, which favors production. The third one is the direct negative effect: By (1), if $D$ is lengthened, the output loss due to the institutional barriers to adoption will increase. Therefore, the total effect on the output level is in general ambiguous. However, for all the parameterizations we try, the total effect is systematically negative. According to Greenwood-Yorokoglu (1997), adoption costs may amount to about $10 \%$ of GDP in developed countries. We have chosen our parametrizations so that the ratio adoption costs to production, $\frac{\int_{t-D}^{t} \alpha H(s) e^{\gamma s} d s}{Y(t)}$, does not exceed $10 \%$ in the long run.

Figure 1 gives the output level in terms of the adoption period for various values of $\alpha$ while $r=0.05, \gamma=0.03$ and $\beta=0.5$. The steady value for output level appears clearly decreasing in $D$. Hence, for admissible parameterizations, the positive effect 


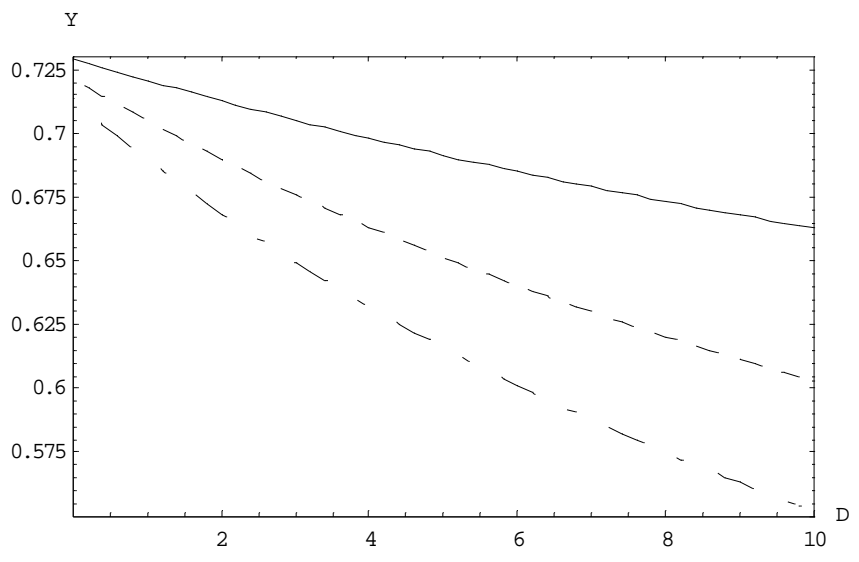

Figure 1. $-\alpha=0.1,--\alpha=0.2,-.-\alpha=0.3$

arising from the increase in the lifetime of the production units is not sufficient to compensate the negative effects inherent to adoption costs.

\section{Short run fluctuations and convergence}

Using (3) and (9), we can eliminate $U(t)$ and obtain an integral equation involving only $H(t)$. Differentiating the obtained equation and (1), we get

$$
\begin{aligned}
H^{\prime}(t) & =-\frac{1-\beta}{\beta} e^{-\gamma T^{*}}\left[H(t)-H\left(t-T^{*}\right)\right] \\
Y^{\prime}(t) & =e^{\gamma t}\left[(1-\alpha) H(t)+\alpha H(t-D) e^{-\gamma D}-H\left(t-T^{*}\right) e^{-\gamma T^{*}}\right]
\end{aligned}
$$

with the initial conditions

$$
\begin{aligned}
& H(0)=\frac{1-\beta}{\beta} e^{-\gamma T^{*}}\left[1-\int_{-T^{*}}^{0} H(s) d s\right] \\
& Y(0)=\int_{-T^{*}}^{0} H(s) e^{\gamma s} d s-\alpha \int_{-D}^{0} H(s) e^{\gamma s} d s .
\end{aligned}
$$

We get a system of two differential equations with two delays $T^{\star}$ and $D$ (see Bellman and Cooke (1963) for a deep introduction into delay differential equations). Note that we keep the same differential structure in the absence of adoption costs, in such a case only delay, $T^{*}$ is involved as it can be inferred from the previous system once $\alpha=D=0$. We solve the general delay differential system using the explicit method of steps developed in Boucekkine, Licandro and Paul (1997). In all our experiments, we obtain oscillatory convergence to the steady state, reflecting the "echo principle" at work in such vintage models. Our findings can be summarized as follows. First, a rise in the adoption costs through $D$ or/and $\alpha$ increases the magnitude and the 


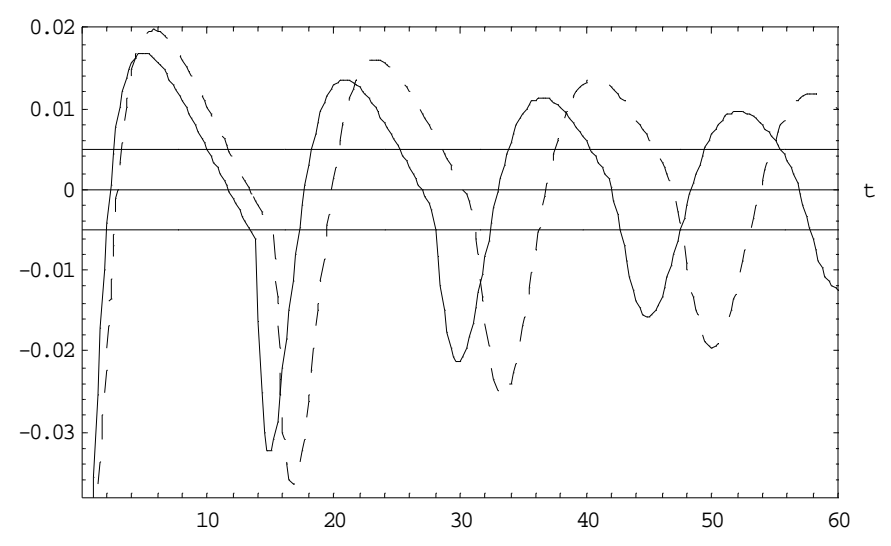

Figure 2. $-D=0,--D=2$

length of the short run fluctuations. Clearly, if $D$ increases, the optimal scrapping time will also increase, and this affects directly the frequency of the echo oscillations. However, adoption costs are also found to involve stronger responses of investment and output to initial deviations with respect to the steady state. Figure 2 provides the short run fluctuations of two economies, starting $10 \%$ below their respective long run output level. The first economy has no institutional barrier to adoption $(\alpha=D=0)$ and the second faces such a constraint with $\alpha=0.2$ and $D=2$ years. $^{4}$

Clearly, the deviations with respect to the respective steady state values are larger in the presence of adoption costs. The cycles are longer and larger in the latter case. As a consequence, the convergence is definitely slower. In our example, the deviation with respect to the steady state is lower than $0.5 \%$ after 120 years for the first economy and around 170 years for the economy with adoption costs. The difference is huge! Our model predicts slower convergence speeds for increasing adoption costs. It also implies that the economic cycles are shorter and smoother for the economies with low adoption costs.

\section{Conclusion}

In this paper, we analyze a simple extension of a canonical creative destruction model including adoption costs. If we believe that adoption costs are highly (negatively) correlated with economic development, our simple model implies that the economic cycles should be much less smoother and much longer in developing countries. It also predicts much slower convergence speed as compared with the model without adoption cost. It would be highly interesting to test the robustness of our predictions in more sophisticated models, including notably the human capital requirement to technology adoption.

\footnotetext{
$\overline{{ }^{4} \text { For both economies, we set } r=0} .05, \gamma=0.03$ and $\beta=0.5$.
} 


\section{References}

Bellman, R. and L. Cooke, 1963, Differential-difference equations (Academic Press, New York).

Benhabib, J. and A. Rustichini, 1991, Vintage capital, investment and growth, Journal of Economic Theory 55, 323-339.

Boucekkine, R., F. Del Río and O. Licandro, 1999, Endogenous Vs exogenously driven fluctuations in vintage capital models, Journal of Economic Theory 88, 161-187.

Boucekkine, R., M. Germain and O. Licandro, 1997, Replacement echoes in the vintage capital growth model, Journal of Economic Theory 74, 333-348.

Boucekkine, R., O. Licandro and C. Paul, 1997, Differential-difference equations in economics: On the numerical solution of vintage capital growth models, Journal of Economic Dynamics and Control 21, 347-362.

Caballero, R. and M. Hammour, 1996, On the timing and efficiency of creative destruction, Quarterly Journal of Economics 111 , 805-851.

Greenwood, J. and M. Yorokoglu, 1997, 1974, Carnegie-Rochester Series on Public Policies 46, 49-95.

Jovanovic, B., 1996, Learning and growth, in D. Kreps and K. Wallis, eds., Advances in economics (Cambridge University Press, London).

Parente, S., 1997, The Problem of economic development, Revista Brasileira de Economia 51, 415-445.

Parente, S., 1994, Technology adoption, learning by doing, and economic growth, Journal of Economic Theory 63, 346-369.

Parente, S and E. Prescott, 1994, Barriers to technology adoption and development, Journal of Political Economy 102, 298-321. 